PROCEEDINGS OF THE

AMERICAN MATHEMATICAL SOCIETY

Volume 135, Number 8, August 2007, Pages 2365-2367

S 0002-9939(07)08843-0

Article electronically published on March 22, 2007

\title{
NON-ABELIAN LOCAL INVARIANT CYCLES
}

\author{
YEN-LUNG TSAI AND EUGENE Z. XIA
}

(Communicated by Michael Stillman)

\begin{abstract}
Let $f$ be a degeneration of Kähler manifolds. The local invariant cycle theorem states that for a smooth fiber of the degeneration, any cohomology class, invariant under the monodromy action, comes from a global cohomology class. Instead of the classical cohomology, one may consider the non-abelian cohomology. This note demonstrates that the analogous nonabelian version of the local invariant cycle theorem does not hold if the first non-abelian cohomology is the moduli space (universal categorical quotient) of the representations of the fundamental group.
\end{abstract}

A degeneration of Kähler manifolds is a proper map $f$ from a Kähler manifold $X$ onto the unit disk $\Delta$ such that $f$ is of maximum rank for all $s \in \Delta$ except at the point $s=0$. Let $\Delta^{*}=\Delta-\{0\}$. We call $X_{t}=f^{-1}(t)$ a smooth fiber or generic fiber when $t \in \Delta^{*}$, and $X_{0}=f^{-1}(0)$ the singular or degenerated fiber. We assume $X_{0}$ has normal crossing singularities.

Fix $t \in \Delta^{*}$ and a base point $x \in X_{t}$ once and for all. There is a monodromy action (see, for example, [3] )

$$
\pi_{1}\left(\Delta^{*}\right) \times \mathrm{H}^{n}\left(X_{t}, \mathbb{C}\right) \rightarrow \mathrm{H}^{n}\left(X_{t}, \mathbb{C}\right) .
$$

The local invariant cycle theorem states that a cohomology class in $\mathrm{H}^{n}\left(X_{t}, \mathbb{C}\right)$, fixed by the monodromy action, is a restriction of a cohomology class in $\mathrm{H}^{n}(X, \mathbb{C})$ 2, 9, 10. Fix a generator $T \in \pi_{1}\left(\Delta^{*}\right) \cong \mathbb{Z}$. Then $T$ determines the monodromy action and gives rise to the isomorphisms

$$
T^{*}: \mathrm{H}^{n}\left(X_{t}, \mathbb{C}\right) \rightarrow \mathrm{H}^{n}\left(X_{t}, \mathbb{C}\right), \quad T_{*}: \pi_{1}\left(X_{t}, x\right) \rightarrow \pi_{1}\left(X_{t}, y\right) .
$$

These isomorphisms are actually induced from a Picard-Lefschetz diffeomorphism which we shall also denote by

$$
T: X_{t} \rightarrow X_{t}
$$

where $y=T(x)$.

The map $f$ induces a strong deformation retraction $X \rightarrow X_{0}$, hence, also an isomorphism $\mathrm{H}^{n}\left(X_{0}, \mathbb{C}\right) \rightarrow \mathrm{H}^{n}(X, \mathbb{C})[2,3$, 6]. Define

$$
c: X_{t} \hookrightarrow X \rightarrow X_{0},
$$

Received by the editors December 6, 2004 and, in revised form, April 18, 2006.

2000 Mathematics Subject Classification. Primary 14D05, 20F34, 55N20.

Tsai is partially supported by the National Center for Theoretical Sciences, Hsinchu, Taiwan; Xia gratefully acknowledges partial support by National Science Council Taiwan grant NSC 932115-M-006-002.

(C)2007 American Mathematical Society Reverts to public domain 28 years from publication 
where the first map is the inclusion and the second the strong deformation retraction. Then $c$ induces homomorphisms:

$$
c^{*}: \mathrm{H}^{n}\left(X_{0}, \mathbb{C}\right) \rightarrow \mathrm{H}^{n}\left(X_{t}, \mathbb{C}\right), \quad c_{*}: \pi_{1}\left(X_{t}, x\right) \rightarrow \pi_{1}\left(X_{0}, c(x)\right) .
$$

The local invariant cycle theorem states that [2, 9, 10]

$$
\operatorname{im}\left(c^{*}\right)=\left\{\alpha \in \mathrm{H}^{n}\left(X_{t}, \mathbb{C}\right) \mid T^{*}(\alpha)=\alpha\right\} .
$$

To generalize the above statement to the non-abelian context, we choose the universal categorical quotient

$$
\mathrm{H}^{1}\left(X_{s}, G\right)=\operatorname{Hom}\left(\pi_{1}\left(X_{s}, x\right), G\right) / / G, \quad s \in \Delta,
$$

as the model for the first non-abelian Betti cohomology on $X_{s}$ with coefficients in the algebraic group $G$. Here $G$ acts on $\operatorname{Hom}\left(\pi_{1}\left(X_{s}, x\right), G\right)$ by conjugation [4, 5]. For any $\rho \in \operatorname{Hom}\left(\pi_{1}\left(X_{t}, x\right), G\right)$, denote by $[\rho]$ its image in $\mathrm{H}^{1}\left(X_{t}, G\right)$ by the universal morphism.

The map $T_{*}$ induces a morphism

$$
T^{\#}: \operatorname{Hom}\left(\pi_{1}\left(X_{t}, T(x)\right), G\right) \rightarrow \operatorname{Hom}\left(\pi_{1}\left(X_{t}, x\right), G\right)
$$

defined by

$$
T^{\#}(\rho)(A)=\rho \circ T_{*}^{-1}(A)
$$

for all $A \in \pi_{1}\left(X_{t}, x\right)$. Similarly, $c$ induces a map

$$
c^{\#}: \operatorname{Hom}\left(\pi_{1}\left(X_{0}, c(x)\right), G\right) \rightarrow \operatorname{Hom}\left(\pi_{1}\left(X_{t}, x\right), G\right)
$$

defined by

$$
c^{\#}(\rho)(A)=\rho \circ c_{*}(A) .
$$

The maps $T^{\#}$ and $c^{\#}$ descend to morphisms on their respective universal categorical quotients:

$$
T^{\#}: \mathrm{H}^{1}\left(X_{t}, G\right) \rightarrow \mathrm{H}^{1}\left(X_{t}, G\right), \quad c^{\#}: \mathrm{H}^{1}\left(X_{0}, G\right) \rightarrow \mathrm{H}^{1}\left(X_{t}, G\right) .
$$

In this setting, the analogous statement of the local invariant cycle theorem is

$$
\operatorname{im}\left(c^{\#}\right)=\left\{[\rho] \in \mathrm{H}^{1}\left(X_{t}, G\right) \mid T^{\#}([\rho])=[\rho]\right\} .
$$

Notice that when $G=\mathbb{C}$ (the addition group), $\mathrm{H}^{1}\left(X_{s}, G\right)$ is the regular first cohomology $\mathrm{H}^{1}\left(X_{s}, \mathbb{C}\right)$ of $X_{s}$ for $s \in \Delta$ and (2) reduces to the classical local invariant cycle theorem (11).

Theorem 1. For each $g>1$, there exists an $n$ and a degeneration $f: X \rightarrow \Delta$ with the generic fiber $X_{t}$ a Riemann surface of genus $g$ and such that

$$
\operatorname{im}\left(c^{\#}\right) \neq\left\{[\rho] \in \mathrm{H}^{1}\left(X_{t}, G\right) \mid T^{\#}([\rho])=[\rho]\right\},
$$

where $G=\operatorname{SL}(n, \mathbb{C})$. More precisely, for such an $n$, there exists an irreducible representation $\rho \in \operatorname{Hom}\left(\pi_{1}\left(X_{t}, x\right), G\right)$ with $\operatorname{im}(\rho) \subset \mathrm{SU}(n)$ and $[\rho] \in\left\{[\rho] \in \mathrm{H}^{1}\left(X_{t}, G\right) \mid\right.$ $\left.T^{\#}([\rho])=[\rho]\right\}$ but $[\rho] \notin \operatorname{im}\left(c^{\#}\right)$.

Proof. For each $n$, let $G=\mathrm{SL}(n, \mathbb{C})$ and $K=\mathrm{SU}(n)$. Let $\Gamma$ be the mapping class group of $X_{t}$. Then each $\gamma \in \Gamma$ induces a map

$$
\gamma: \operatorname{Hom}\left(\pi_{1}\left(X_{t}, \gamma(x)\right), G\right) \rightarrow \operatorname{Hom}\left(\pi_{1}\left(X_{t}, x\right), G\right): \quad \gamma(\rho)(A)=\rho \circ \gamma^{-1}(A) .
$$

These maps provide a $\Gamma$-action on $\mathrm{H}^{1}\left(X_{t}, G\right)$. In [1, Andersen proved that for $g>1$, there exists infinitely many $n$ such that there exists irreducible $\rho \in \operatorname{Hom}\left(\pi_{1}\left(X_{t}\right), G\right)$ with $\operatorname{im}(\rho) \subset K$ and $\gamma([\rho])=[\rho]$ for all $\gamma \in \Gamma$. 
Now suppose our choices of $\rho$ and $n$ satisfy the hypothesis and conclusions of Andersen's theorem [1]. Then since $T: X_{t} \rightarrow X_{t}$ is a diffeomorphism, $T_{*} \in \Gamma$. Hence $T^{\#}([\rho])=[\rho]$.

The fundamental group $\pi_{1}\left(X_{t}, x\right)$ has a presentation

$$
\left\langle A_{i}, 1 \leq i \leq 2 g, \mid \prod_{i=1}^{g} A_{i} A_{g+i} A_{i}^{-1} A_{g+i}^{-1}\right\rangle,
$$

where the $A_{i}$ 's correspond to simple loops beginning and ending at $x$. Since $\rho$ is an irreducible representation, $\rho\left(A_{i}\right) \neq I \in G$ for some $i$.

Now let $f: X \rightarrow \Delta$ be a degeneration with $A_{i}$ as its vanishing cycle. Then $c_{*}\left(A_{i}\right)=e \in \pi\left(X_{0}, c(x)\right)$. Hence for every $[\alpha] \in \mathrm{H}^{1}\left(X_{0}, G\right)$,

$$
c^{\#}(\alpha)\left(A_{i}\right)=\alpha\left(c_{*}\left(A_{i}\right)\right)=I \in G .
$$

Since $\rho\left(A_{i}\right) \neq I,[\rho] \neq[\alpha]$ for all $[\alpha] \in \operatorname{im}\left(c^{\#}\right)$.

Final remarks. We refer to 1 for the detailed and explicit descriptions of Andersen's irreducible representation classes fixed by the $\Gamma$-action. Here we simply mention that all such representations have finite images in $\mathrm{SU}(n)$. It will be interesting to see whether there exist examples $[\rho]$, satisfying the conclusion of Theorem[1, with $\operatorname{im}(\rho)$ being Zariski dense in $G$. Unfortunately, such representations are unlikely to satisfy the overly stringent condition of being fixed by the $\Gamma$-action. In fact, it is likely that the $\Gamma$-orbit of $[\rho]$ is Zariski dense if $\operatorname{im}(\rho)$ is Zariski dense in $G$. This is the case when $G=\mathrm{SL}(2, \mathbb{C}), K=\mathrm{SU}(2)$ [7, 8].

\section{REFERENCES}

[1] Jørgen Ellegaard Andersen, Fixed points of the mapping class group in the $\mathrm{SU}(n)$ moduli spaces, Proc. Amer. Math. Soc. 125 (1997), no. 5, 1511-1515. MR1376748 (97j:58014)

[2] C. H. Clemens, Degeneration of Kähler manifolds, Duke Math. J. 44 (1977), no. 2, 215-290. MR0444662 (56:3012)

[3] Vik. S. Kulikov and P. F. Kurchanov, Complex algebraic varieties: periods of integrals and Hodge structures, Algebraic geometry, III, Encyclopaedia Math. Sci., vol. 36, Springer, Berlin, 1998, pp. 1-217, 263-270. MR.1602375

[4] Alexander Lubotzky and Andy R. Magid, Varieties of representations of finitely generated groups, Mem. Amer. Math. Soc. 58 (1985), no. 336, xi+117. MR0818915 (87c:20021)

[5] David Mumford, Geometric invariant theory, Ergebnisse der Mathematik und ihrer Grenzgebiete, Neue Folge, Band 34, Springer-Verlag, Berlin, 1965. MR0214602 (35:5451)

[6] Ulf Persson, On degenerations of algebraic surfaces, Mem. Amer. Math. Soc. 11 (1977), no. $189, \mathrm{xv}+144$. MR0466149 (57:6030)

[7] Joseph P. Previte and Eugene Z. Xia, Topological dynamics on moduli spaces. I, Pacific J. Math. 193 (2000), no. 2, 397-417. MR1755824 (2001e:53090)

[8] _ Topological dynamics on moduli spaces. II, Trans. Amer. Math. Soc. 354 (2002), no. 6, 2475-2494 (electronic). MR.1885660(2002k:53167)

[9] Wilfried Schmid, Variation of Hodge structure: the singularities of the period mapping, Invent. Math. 22 (1973), 211-319. MR0382272 (52:3157)

[10] Joseph Steenbrink, Limits of Hodge structures, Invent. Math. 31 (1975/76), no. 3, 229-257. MR0429885 (55:2894)

Department of Mathematical Sciences, National Chengchi University, Taipei 116, TAIWAN

E-mail address: yenlung@math.nccu.edu.tw

Department of Mathematics, National Cheng Kung University, Tainan 701, Taiwan

E-mail address: ezxia@ncku.edu.tw 